\title{
An Epidemic Model With Post-Contact Prophylaxis of Distributed Length II. Stability and Oscillations if Treatment is Fully Effective
}

\author{
H. R. Thieme ${ }^{1}$, A. Tridane, and Y. Kuang \\ Department of Mathematics and Statistics, Arizona State University \\ Tempe, AZ 85287-1804, U.S.A.
}

\begin{abstract}
A possible control strategy against the spread of an infectious disease is the treatment with antimicrobials that are given prophylactically to those that had contact with an infective person. The treatment continues until recovery or until it becomes obvious that there was no infection in the first place. The model considers susceptible, treated uninfected exposed, treated infected, (untreated) infectious, and recovered individuals. The overly optimistic assumptions are made that treated uninfected individuals are not susceptible and treated infected individuals are not infectious. Since treatment lengths are considered that have an arbitrary distribution, the model system consists of ordinary differential and integral equations. We study the impact of the treatment length distribution on the large-time behavior of the model solutions, namely whether the solutions converge to an equilibrium or whether they are driven into undamped oscillations.
\end{abstract}

Key words: basic reproduction number, standard incidence, (class) age structure, distributed time delay, disease persistence, global stability of endemic equilibria, instability, periodic solutions, frequency domain

AMS subject classification: 34K20, 37N25, 92C50, 92D25, 92D30

\section{Introduction}

Antimicrobial drugs can be useful for the control and treatment of infections, in particular when vaccination is not possible or effective or cannot be administered in time. The best-known example are antibiotics which are used against bacterial diseases. Examples of antiviral drugs against in-

${ }^{1}$ Corresponding author. E-mail: h.thieme@ asu.edu 
fluenza are amantadines which are only effective against influenza A and neuraminidase inhibitors which are effective against influenza A and B (see [8] for details). If antimicrobial treatment is only applied after an infected individual develops symptoms, we speak about symptomatic treatment. Treatment can also be prophylactic. In this case the drugs are administered to individuals that have been exposed to the disease by contact with an infective individual. If infection has occurred, the treatment supposedly reduces the duration and severity of infectiousness; if not, the treatment supposedly reduces the susceptibility in case of another exposure.

This study focuses on the effect of prophylactic treatment on the dynamics of an infectious disease. Our model is a simplification of the one studied in [8]. Since we focus on prophylactic treatment, we ignore symptomatic treatment and consequently do not distinguish between symptomatic and asymptomatic infectives (though in reality their infectiousness may be different [1, Tab.1]). We need to distinguish between treated and untreated infected individuals though. In order to keep the analysis from becoming too complicated, we make the overly optimistic assumption that treated infected individuals are not infectious. (In [1, Tab.1] the rate of infection by treated symptomatic individuals is assumed to be 0.4 that by untreated symptomatic individuals, and in [8, Tab.A1] the rate of infection by treated wild-type virus has been estimated to be 0.5 that by untreated wild-type virus.) Since prophylactic treatment is used before infection is confirmed, we need to consider a class of uninfected exposed but treated individuals, a novel feature introduced in [8]. Notice that we use the term 'exposed' with the meaning of having had a contact with an infectious individual whether or not an infection has actually occurred. (In mathematical epidemiology, 'exposed' has been often used with the meaning 'infected, but not yet infectious, i.e. in the latent stage'.)

In a prequel to this paper [18], we have derived a threshold condition for the treatment to be able to eradicate the disease. This threshold has been formulated in terms of a basic replacement ratio (disease reproduction number), $\mathcal{R}_{0}$, that gives the expected number of secondary infections which one average infected individual produces when introduced into an otherwise completely susceptible population. If $\mathcal{R}_{0} \leq 1$, i.e. if an infected individual can hardly replace itself, the disease dies out. The threshold condition is sharp, as we have shown for $\mathcal{R}_{0}>1$ that the disease persists uniformly in the host population (Theorem 1...3), in the sense that the number of infectives is bounded away from 0 for large times (with the asymptotic bound not depending on the initial conditions). In this paper, we will study whether prophylactic treatment drives the disease towards an endemic equilibrium or rather into undamped oscillations in case that eradication cannot be achieved.

More generally than in [8], we will consider an arbitrary distribution for how long uninfected exposed individuals are treated (in case that they are treated). In [8], an exponential distribution is assumed, i.e. treatment is terminated at a fixed rate (which in [8] may depend on chronological age). The other extreme is a treatment of fixed length which will be also included in our model. The impact of the distribution of stage durations on disease dynamics has been studied for the recovery (immune) period [10, 15, 17] and for a period of isolation (quarantine) [7]. In this paper, it will be investigated for the duration of prophylactic treatment. In order to cope with arbitrary length distributions of treatment, we stratify the treated exposed but uninfected individuals according to their treatment age (the time that has passed since treatment started). This way it becomes an age- 
structured model. A large class of age-structured models has been studied by Glenn Webb in many papers and in his monograph [19]. Under the most general assumptions, our model will not fit into this class; still the semigroup methods developed in [19] will be useful.

In order to keep the analytic complexity at bay, we will make a second overly optimistic assumption, namely that treatment is fully effective also in so far that treated uninfected individuals are not susceptible. (In [8, Tab.A1], the susceptibility of treated uninfected individuals has been estimated to be 0.5 that of susceptible individuals.) This will allow us to reduce the model to a single Stieltjes-integro-differential equation and apply powerful frequency-domain (i.e. Fourier analysis) techniques [11].

\section{Contents}

1. The model, existence of solutions, and disease persistence 269

2. Stability and oscillations: the results 274

$\begin{array}{ll}\text { 3. Discussion } & 279\end{array}$

$\begin{array}{lll}\text { A Proofs } & 281\end{array}$

A1. Transformation to a single equation . . . . . . . . . . . . . 281

A2. Local stability of the endemic equilibrium . . . . . . . . . . . . 285

A3. Global stability of the endemic equilibrium . . . . . . . . . . . . . 287

A4. Some frequency domain results . . . . . . . . . . . . . . . . . 288

A5. Instability of the endemic equilibrium . . . . . . . . . . . . . 289

\section{The model, existence of solutions, and disease persistence}

As another simplification, we assume that births balance deaths and that nobody dies from the disease. Consequently, the size of the host population remains constant. The proportion of susceptible individuals, at time $t$, is denoted by $S(t)$, the proportion of uninfected exposed but treated individuals by $U(t)$, the proportion of infective (and untreated) individuals by $I(t)$, the proportion of treated infected individuals by $J(t)$ and the proportion of recovered individuals by $R(t)$ (see Table 1.),

$$
1=S(t)+U(t)+I(t)+J(t)+R(t) .
$$

Susceptible individuals (S-class) that are exposed (i.e. have a contact with an infective individual) move into the U-class if they are not infected but treated (see Figure 1.). If they are infected and treated, they move into the J-class. If they are infected and not treated, they move into the I-class. U-individuals (uninfected treated) become susceptible again (move back into the S-class) once treatment is terminated. Infected individuals, whether in the I-class (treated) or in the J-class 


\begin{tabular}{|cl|}
\hline$t$ & time \\
$a$ & treatment age \\
$S(t)$ & proportion of susceptibles at time $t$ \\
$U(t)$ & proportion of treated uninfected exposed individuals at time $t$ \\
$u(t, \cdot)$ & treatment age density of treated uninfected exposed individuals \\
$I(t)$ & proportion of infectives at time $t$ \\
$J(t)$ & proportion of treated infected individuals at time $t$ \\
$R(t)$ & proportion of removed individuals at time $t$ \\
\hline
\end{tabular}

Table 1: Model variables

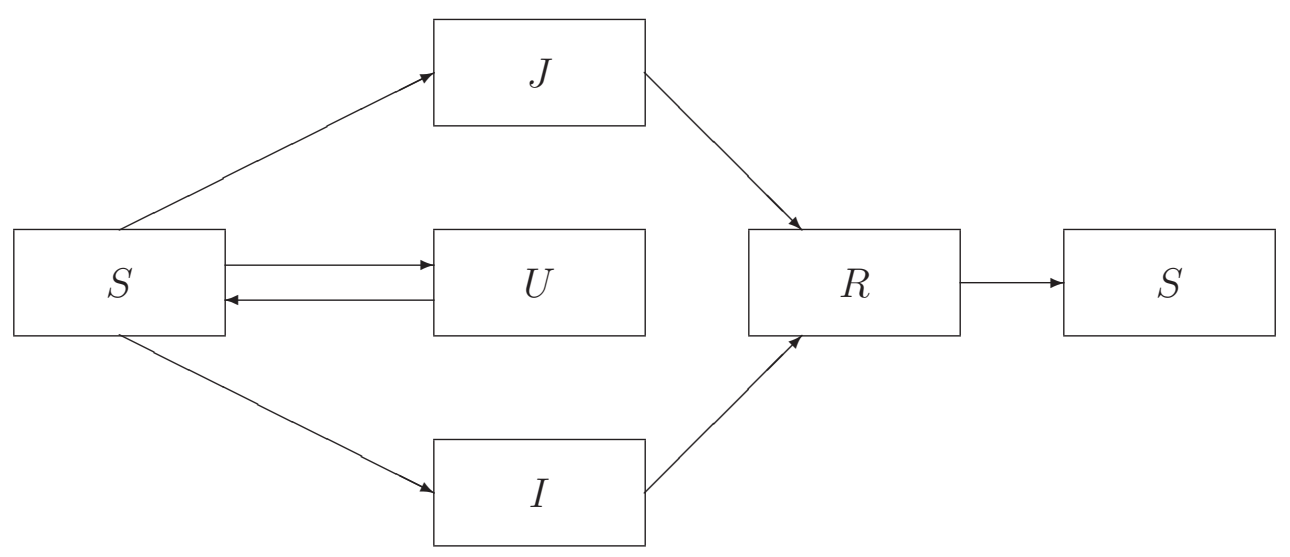

Figure 1: Flow diagram of the model.

(untreated), recover into the R-class where they are immune to the disease. R-individuals, upon losing their immunity, return to the S-class.

Exposed individuals, whether infected or not, are treated to the degree possible. For mathematical simplicity, we make the overly optimistic assumption that infected individuals that are treated (denoted by $J$ ) are not infective. We also assume that the treatment of infected individuals continues until they have recovered from the disease. Differently from [18] where we assume that treatment reduces the susceptibility of a treated exposed, but uninfected individual, we make another even more optimistic assumption, namely that an exposed uninfected individual is not susceptible to the disease at all as long as it is treated. Ignoring a latency period, we further assume that untreated infected individuals are infectious. Untreated infected individuals recover from the disease at a fixed rate $\gamma_{1}$, treated infected individuals at a fixed rate $\gamma_{2}$.

We let $p$ denote the probability that a susceptible individual is actually infected upon the contact with an infective individual and $\tau$ the probability that an exposed individual (whether infected or not) is treated. If $\kappa$ is the per capita contact rate, the incidence is $p \kappa I S$. We have the following 
equations,

$$
\begin{aligned}
I^{\prime} & =(1-\tau) p \kappa I S-\left(\mu+\gamma_{1}\right) I, \\
J^{\prime} & =\tau p \kappa I S-\left(\mu+\gamma_{2}\right) J, \\
R^{\prime} & =\gamma_{1} I+\gamma_{2} J-(\mu+\rho) R, \\
S & =1-U-I-J-R,
\end{aligned}
$$

The natural per capita death rate $\mu$ is also the per capita birth rate and $\rho$ is the rate at which recovered individuals become susceptible again. All parameters are non-negative. Typically the natural death rate $\mu$ is much smaller than the other rates and one may like to consider the limiting case $\mu=0$; then $\gamma_{1}, \gamma_{2}$ and $\rho$ are assumed to be positive.

\begin{tabular}{|cl|}
\hline$\mu$ & per capita mortality and birth rate \\
$\gamma_{1}$ & p. cap. recovery rate of (untreated) infectious individuals \\
$\gamma_{2}$ & p. cap. recovery rate of treated infected individuals \\
$\rho$ & per capita rate at which a recovered individual looses immunity \\
$\kappa$ & per capita contact rate \\
$p$ & infection probability at a contact \\
$\tau$ & probability that an exposed individual is treated \\
\hline
\end{tabular}

Table 2: Parameters

The uninfected exposed and treated individuals, denoted by $U$, are stratified along their treatment age, $a$, the time that has passed since their treatment started,

$$
U(t)=\int_{0}^{\infty} u(t, a) d a
$$

where $u(t, \cdot)$ is the treatment age density of uninfected exposed and treated individuals at time $t$. In deriving a formula for $u(t, a)$ we must distinguish the cases $t<a$ and $t>a$. For $t>a, u(t, a)$ describes the treated uninfected individuals that were exposed after the beginning, namely at time $t-a>0$, at rate $\kappa S(t-a) I(t-a)$, and so

$$
u(t, a)=\tau(1-p) \kappa S(t-a) I(t-a) \mathcal{G}(a), \quad t>a .
$$

Here $1-p$ is the probability of having not been infected at exposure. Further

$$
\mathcal{G}(a)=e^{-\mu a} \mathcal{F}(a),
$$

where $e^{-\mu a}$ is the probability of not having died a natural death in a time span of length $a$ and $\mathcal{F}(a)$ is the probability of still being treated at treatment age $a$. So $\mathcal{G}(a)$ is the probability of being still in the class of treated exposed though uninfected individuals. 
For $a>t \geq 0, u(t, a)$ describes the uninfected individuals that had been exposed before time 0 and had exposure age $a-t$ at time 0 ,

$$
u(t, a)=u_{0}(a-t) \frac{\mathcal{G}(a)}{\mathcal{G}(a-t)}, \quad a>t
$$

Here $\frac{\mathcal{G}(a)}{\mathcal{G}(a-t)}$ is the conditional probability of still being in the class of treated though uninfected individuals at exposure age $a$ under the proviso of having been in this class at time 0 , with exposure age $a-t$. The class age density $u_{0}$ represents the individuals in the treated exposed though uninfected class at time $0, \int_{0}^{\infty} u_{0}(a) d a=U(0)$.

We assume that $\mathcal{F}: \mathbb{R}_{+} \rightarrow \mathbb{R}$ is non-negative and monotone decreasing. We assume that $D_{T}=\int_{0}^{\infty} \mathcal{F}(a) d a$, the average time an exposed individual is treated (neglecting natural death) [16, Ch.12], is finite. $\tilde{D}_{T}=\int_{0}^{\infty} \mathcal{G}(a) d a$ is the average duration of treatment for exposed individuals taking natural death into account. We summarize,

$$
\begin{aligned}
U(t) & =\int_{0}^{\infty} u(t, a) d a, \\
u(t, a) & =\left\{\begin{array}{cc}
\tau(1-p) \kappa S(t-a) I(t-a) \mathcal{G}(a), & t>a, \\
u_{0}(a-t) \frac{\mathcal{G}(a)}{\mathcal{G}(a-t)}, & a>t,
\end{array}\right.
\end{aligned}
$$

with

$$
\mathcal{G}(a)=e^{-\mu a} \mathcal{F}(a)
$$

To put (1..7) into another perspective, we assume that $\mathcal{F}(a)>0$ for all $a \geq 0$ and that $\mathcal{F}$ is continuously differentiable. Then $\zeta(a)=-\frac{\mathcal{F}^{\prime}(a)}{\mathcal{F}(a)}$ is the rate at which the treatment is terminated $a$ time units after exposure. Assume that $I, S$ and $u_{0}$ are continuously differentiable as well. By $(1 . .7)$

$$
\left.\begin{array}{rl}
\left(\partial_{t}+\partial_{a}\right) u(t, a) & =-(\zeta(a)+\mu) u(t, a), \quad t \neq a \\
u(t, 0) & =\tau(1-p) \kappa S(t) I(t) \\
u(0, a) & =u_{0}(a)
\end{array}\right\} t, a \geq 0 .
$$

Here $\partial_{t} u$ and $\partial_{a} u$ denote the partial derivatives of $u$ with respect to $t$ and $a$ respectively. Integrating this first order PDE with initial and boundary values along characteristics retrieves (1..6) and (1..4). Notice that $\mathcal{F}(a)=\exp \left(-\int_{0}^{a} \zeta(s) d s\right)$. If $\zeta$ and $q$ do not depend on $a$, we can integrate the partial differential equation in (1..9) over $a$ and obtain (at least formally) a differential equation for $U$,

$$
U^{\prime}=(1-p) \kappa S I-(\zeta+\mu) U
$$

In this special case, our model becomes a system of ordinary differential equations in the dependent variables $U, I, R$ with the auxiliary variable $S$. If $\mathcal{F}$ is not differentiable, our model does not quite fit into the framework of [19]. But the methods developed in [19, Sec.4.3] will be still useful in studying the local stability of equilibria. 
Existence and uniqueness of solutions. The system (1..2), (1..7) is a special case of the one in [18] where we have proved the following existence and uniqueness result.

1..1 Theorem. Let $u_{0} \in L_{+}^{1}\left(\mathbb{R}_{+}\right)$and $I_{0}, J_{0}, R_{0} \geq 0$ with $\int_{0}^{\infty} u_{0}(a) d a+I_{0}+J_{0}+R_{0} \leq 1$. Then there exists a unique non-negative continuous solution $u(t, \cdot) \in L^{1}\left(\mathbb{R}_{+}\right), I(t), J(t), R(t) \in \mathbb{R}_{+}$ of (1..2) and (1..7) with initial data $u_{0}, I_{0}, J_{0}$ and $R_{0}$, which is defined for all $t \geq 0$ and satisfies $\int_{0}^{\infty} u(t, a) d a+I(t)+J(t)+R(t) \leq 1$.

Expected remaining length of treatment. We mentioned the special case that treatment is terminated at a fixed rate $\zeta$. Another special case is the one of a fixed length of treatment or, somewhat more generally, a finite maximum length of treatment, $c \in(0, \infty)$. Then $\mathcal{F}(a)>0$ for $a \in(0, c)$ and $\mathcal{F}(a)=0$ for $a>c$. If $\mathcal{F}(a)>0$ for all $a \geq 0$, we set $c=\infty$. An important concept is the expected length of remaining treatment at treatment age $a \in[0, c)$,

$$
D_{T}(a)=\int_{0}^{\infty} \frac{\mathcal{F}(a+t)}{\mathcal{F}(a)} d t, \quad a \in[0, c) .
$$

See [16, Sec.12.4]. If $c<\infty, D_{T}(a) \leq c-a$ [16, Prop.12.4]. If there is a fixed rate at which treatment is terminated, $D_{T}(a)$ is independent of $a$. Throughout the rest of the paper we assume the following.

Overall assumption. We assume that $\mu>0$, or $c<\infty$, or (if $c=\infty$ ) $D_{T}(a)$ is a bounded function of $a \in[0, \infty)$.

In the last case an important estimate holds [16, Prop.12.1].

1..2 Lemma. If $c=\infty$, there exist $\varepsilon>0, M \geq 1$ such that $\frac{\mathcal{F}(a+t)}{\mathcal{F}(a)} \leq M e^{-\varepsilon t}$ for all $t, a \geq 0$.

In any case, by (1..5), there exist constants $M \geq 1, \delta>0$ such that

$$
\frac{\mathcal{G}(a+t)}{\mathcal{G}(a)} \leq M e^{-\delta t}, \quad 0 \leq a<c, t \geq 0 .
$$

Disease persistence versus disease extinction By definition, the disease persist uniformly (strongly), if there exists some $\varepsilon>0$ such that $\liminf _{t \rightarrow \infty} I(t) \geq \varepsilon$ for all solutions with $I(0)>0$. The disease persists uniformly weakly if there exists some $\varepsilon>0$ such that $\lim _{\sup } \operatorname{sum}_{t \rightarrow \infty} I(t) \geq \varepsilon$ for all solutions with $I(0)>0$. We define

$$
\mathcal{R}_{0}=\frac{(1-\tau) p \kappa}{\mu+\gamma_{1}}
$$

$\mathcal{R}_{0}$ is the basic replacement ratio (basic reproduction number of the disease), i.e. the average number of secondary infections produced by one average infected individual that is introduced into an otherwise completely susceptible population. Indeed, $\frac{1}{\mu+\gamma_{1}}$ is the average duration of the infectious period (average sojourn time in the class of untreated infected individuals) with natural death being taken into account. So $\frac{\kappa}{\mu+\gamma}$ is the number of contacts made by one average infected 
individual. $(1-\tau)$ is the probability that this infected individual is actual infectious and $p$ the probability that an exposed susceptible individual contracts the infection. The following is proved in [18], Theorems 4.2 and 4.3 .

1..3 Theorem. The disease is uniformly strongly persistent if $\mathcal{R}_{0}>1$. If $\mathcal{R}_{0} \leq 1$, the disease goes extinct, $I(t) \rightarrow 0$ as $t \rightarrow \infty$.

\section{Stability and oscillations: the results}

The system (1..2) and (1..7) can be transformed into a single scalar integro-differential equation to which the results in [11] can be applied (see Appendix A1.). The first to use [11] for epidemic models were Stech and Williams [15].

Equilibria. An equilibrium of (1..2) and (1..7) is a time-independent solution of this system. So it is a solution of the algebraic system

$$
\begin{aligned}
& 0=(1-\tau) p \kappa I S-\left(\mu+\gamma_{1}\right) I \\
& 0=\tau p \kappa I S-\left(\mu+\gamma_{2}\right) J \\
& 0=\gamma_{1} I+\gamma_{2} J-(\mu+\rho) R \\
& S=1-U-I-J-R
\end{aligned}
$$

where

$$
\begin{aligned}
U & =\int_{0}^{\infty} u(a) d a, \\
u(a) & =\tau(1-p) \kappa S I \mathcal{G}(a) .
\end{aligned}
$$

Notice that $u$ given by (2..2) automatically satisfies (1..6),

$$
u(a)=u(a-t) \frac{\mathcal{G}(a)}{\mathcal{G}(a-t)}, \quad a>t .
$$

There always exists the disease free equilibrium $S=1, U=I=J=R=0, u(a)=0$. By definition, an endemic equilibrium of (1..2) and (1..7), carrying superscript ${ }^{*}$, satisfies $I^{*}>0$ and is described by the system,

$$
\begin{aligned}
0 & =(1-\tau) p \kappa S^{*}-\left(\mu+\gamma_{1}\right), \\
0 & =\tau p \kappa I^{*} S^{*}-\left(\mu+\gamma_{2}\right) J^{*}, \\
0 & =\gamma_{1} I^{*}+\gamma_{2} J^{*}-(\mu+\rho) R^{*}, \\
S^{*} & =1-U^{*}-I^{*}-J^{*}-R^{*}, \\
U^{*} & =(1-\tau) p \kappa S^{*} I^{*} \tilde{D}_{T}, \\
u^{*}(a) & =\tau(1-p) \kappa S^{*} I^{*} \mathcal{G}(a),
\end{aligned}
$$

where

$$
\tilde{D}_{T}=\int_{0}^{\infty} \mathcal{G}(a) d a
$$

is the expected duration of treatment (natural death included). 
Existence of endemic equilibria. The following threshold result is readily proved and consistent with Theorem 1..3.

2..1 Theorem. There is no endemic equilibrium if $\mathcal{R}_{0} \leq 1$, and a unique endemic equilibrium if $\mathcal{R}_{0}>1$.

For $x=(u, I, J, R)$ with $u \in L^{1}(\mathbb{R}), I, J, R \in \mathbb{R}$, we define $\|x\|=\int_{0}^{\infty}|u(a)| d a+|I|+|J|+$ $|R|$.

2..2 Definition (stability concepts). The endemic equilibrium $x^{*}=\left(u^{*}(\cdot), I^{*}, J^{*}, R^{*}\right)$ is called locally stable if for every $\varepsilon>0$ there exists some $\delta>0$ with the following property:

if $x(t)=(u(t, \cdot), I(t), J(t), R(t))$ is a non-negative solution of (1..2), (1..7) and $\|x(0)\| \leq 1$ and $\left\|x(0)-x^{*}\right\|<\delta$, then $\left\|x(t)-x^{*}\right\|<\varepsilon$ for all $t \geq 0$.

The endemic equilibrium $x^{*}$ is called locally asymptotically stable if it is locally stable and there exists some $\delta>0$ such that the following holds:

if $x(t)=(u(t, \cdot), I(t), J(t), R(t))$ is a non-negative solution of (1..2), (1..7) and $\|x(0)\| \leq 1$ and $\left\|x(0)-x^{*}\right\|<\delta$, then $\left\|x(t)-x^{*}\right\| \rightarrow 0$ as $t \rightarrow \infty$.

The endemic equilibrium $x^{*}$ is called globally attractive if $\left\|x(t)-x^{*}\right\| \rightarrow 0$ for every nonnegative solution $x(t)=(u(t, \cdot), I(t), J(t), R(t))$ of (1..2), (1..7) with $\|x(0)\| \leq 1$ and $I(0)>0$.

The endemic equilibrium is called globally asymptotically stable if it is locally asymptotically stable and globally attractive.

The endemic equilibrium is called unstable if it is not locally stable.

Global stability of the endemic equilibrium. The following result is proved in appendix A.

2..3 Theorem. Assume that

$$
-\left(\gamma_{1}+\mu\right) \Re \int_{0}^{\infty} e^{i s t} \mathcal{G}(t) d t \leq \frac{p}{1-p} \frac{1-\tau}{\tau} \quad \forall s>0 .
$$

Then the endemic equilibrium is globally asymptotically stable.

The condition in this theorem is difficult to interpret biologically. We derive a few special cases which have an easier interpretation. By Lemma A.4, the endemic equilibrium is globally stable, if the length distribution of treatment has a special form.

2..4 Corollary. Let $\mathcal{G}$ be convex (e.g. if $\mathcal{F}$ is convex). Then the endemic equilibrium is globally asymptotically stable.

In particular, the endemic equilibrium is globally asymptotically stable when the treatment is terminated at a fixed rate (resulting in exponentially distributed treatment length).

2..5 Corollary. Assume that the treatment period has exponentially distributed length. Then the endemic equilibrium is globally asymptotically stable. 
The endemic equilibrium is also globally asymptotically stable if the average duration of treatment, $\tilde{D}_{T}=\int_{0}^{\infty} \mathcal{G}(a) d a$, is sufficiently short compared to the average duration of the infectious period, $\tilde{D}_{I}=\frac{1}{\gamma_{1}+\mu}$.

2..6 Corollary. Assume that $\frac{\tau}{1-\tau} \tilde{D}_{T} \leq \frac{p}{1-p} \tilde{D}_{I}$. Then the endemic equilibrium is globally asymptotically stable.

In the following we use the Laplace transform notation $\hat{f}(z)=\int_{0}^{\infty} e^{-z t} f(t) d t$.

2..7 Lemma. Assume that the distribution of treatment has a probability density, i.e.

$$
\mathcal{F}(a)=\int_{a}^{\infty} f(t) d t
$$

where $f: \mathbb{R}_{+} \rightarrow \mathbb{R}$ is non-negative and $\int_{0}^{\infty} f(t) d t=1$. Then $\Re \hat{\mathcal{G}}(-i s)$ has the same sign as

$$
\mu(1-\Re \hat{f}(\mu-i s))+s \Im \hat{f}(\mu-i s) .
$$

In particular, for $s>0, \Re \hat{\mathcal{G}}(-i s)>0$ if $\Im \hat{f}(\mu-i s) \geq 0$. Further, if for a fixed $s>0$, $\Im \hat{f}(\mu-i s)<0$, then $\Re \hat{\mathcal{G}}(-i s)<0$ for sufficiently small $\mu>0$.

Proof. By changing the order of integration (or integrating by parts),

$$
\begin{aligned}
\hat{\mathcal{G}}(-i s) & =\hat{\mathcal{F}}(\mu-i s)=\int_{0}^{\infty} e^{-(\mu-i s) t} \mathcal{F}(t) d t \\
& =\frac{1}{\mu-i s}\left(1-\int_{0}^{\infty} e^{-(\mu-i s) t} f(t) d t\right)=\frac{\mu+i s}{\mu^{2}+s^{2}}(1-\hat{f}(\mu-i s)) .
\end{aligned}
$$

2..8 Corollary. Assume that

$$
\mathcal{F}(a)=\int_{a}^{\infty}(f * g)(t) d t
$$

with

$$
(f * g)(t)=\int_{0}^{t} f(t-s) g(s) d s
$$

and $f, g \in L^{1}\left(\mathbb{R}_{+}\right)$that are non-negative, decreasing and convex. Then the endemic equilibrium is globally asymptotically stable.

Proof.

$$
\int_{0}^{\infty} e^{i s t} \mathcal{G}(t) d t=\int_{0}^{\infty} e^{(i s-\mu) t} \mathcal{F}(t) d t=\frac{\mu+i s}{s^{2}+\mu^{2}}\left(1+\int_{0}^{\infty} e^{(i s-\mu) t} d \mathcal{F}(t)\right)
$$


So $\int_{0}^{\infty} e^{i s t} \mathcal{G}(t) d t$ has nonnegative real part if

$$
0 \leq-\Im \int_{0}^{\infty} e^{(i s-\mu) t} d \mathcal{F}(t)=\Im \int_{0}^{\infty} e^{(i s-\mu) t}(f * g)(t) d t
$$

We use that the Laplace transform of a convolution is the product of the Laplace transforms and the notation $g_{\mu}(t)=e^{-\mu t} g(t)$,

$$
\begin{aligned}
& \Im \int_{0}^{\infty} e^{(i s-\mu) t}(f * g)(t) d t=\Im\left(\int_{0}^{\infty} e^{(i s-\mu) t} f(t) d t \int_{0}^{\infty} e^{(i s-\mu) t} g(t) d t\right) \\
= & \Im\left(\int_{0}^{\infty} e^{i s t} f_{\mu}(t) d t \int_{0}^{\infty} e^{i s t} g_{\mu}(t) d t\right) \\
= & \int_{0}^{\infty} \cos s t f_{\mu}(t) d t \int_{0}^{\infty} \sin s t g_{\mu}(t) d t+\int_{0}^{\infty} \sin s t f_{\mu}(t) d t \int_{0}^{\infty} \cos s t g_{\mu}(t) d t .
\end{aligned}
$$

The product of two non-negative, decreasing and convex functions has the same properties, and so $f_{\mu}$ and $g_{\mu}$ are non-negative, decreasing, and convex. By Lemma A.3 and A.4, all expressions in the last line of the chain of equations are non-negative, and $\int_{0}^{\infty} e^{i s t} \mathcal{G}(t) d t$ has non-negative real part.

Instability of the endemic equilibrium. To a certain degree, the next result is a converse to Theorem 2..3. It is proven in Appendix A5..

2..9 Theorem. Let $\Re \int_{0}^{\infty} e^{i y t} \mathcal{G}(t) d t<0$ for some $y>0$.

Given the parameters $\rho \geq 0, \mu \geq 0, \gamma_{2}>0$, the parameters $0<p<1,0<\tau<1, \gamma_{1}>0$ and $\kappa>0$ can be chosen in such a way that the endemic equilibrium is unstable.

In fact, $p$ and $\kappa$ can be changed in such a way that a root of the characteristic equation crosses the imaginary axis.

Notice that this result also holds for $\rho=0$, the case that immunity is permanent.

Treatment of fixed length. Treatment of fixed length $c$ is modeled by $\mathcal{F}(a)=1$ if $0 \leq a<c$, and $\mathcal{F}(a)=0$ if $a>c$. Since $\mathcal{G}(a)=e^{-\mu a} \mathcal{F}(a)$,

$$
\int_{0}^{\infty} e^{i s t} \mathcal{G}(t) d t=\int_{0}^{c} e^{i s t} e^{-\mu t} d t=\frac{1-e^{-(\mu-i s) c}}{\mu-i s}=\frac{(\mu+i s)\left(1-e^{-(\mu-i s) c}\right)}{\mu^{2}+s^{2}}
$$

and its real part has the same sign as $\mu\left(1-e^{-\mu c} \cos (s c)\right)+s e^{-\mu c} \sin s c$. If $\mu$ is small as it realistically is, we can find some $s>0$ such that $\Re \int_{0}^{\infty} e^{i y t} \mathcal{G}(t) d t<0$. Theorem $2 . .9$ implies the following result. 


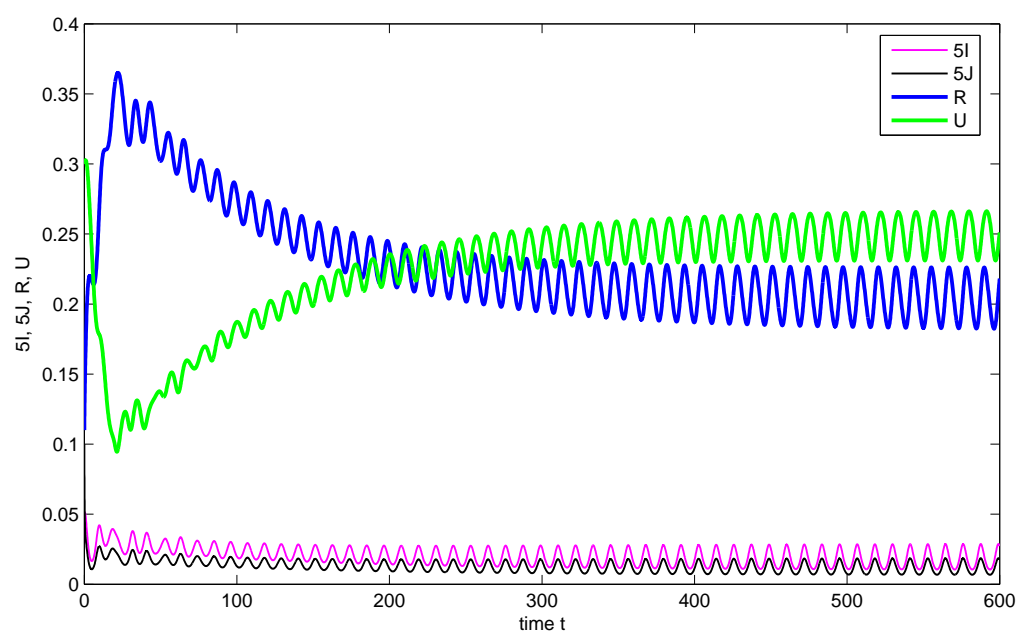

Figure 2: Approach to a periodic solution for treatment of fixed length. Here $\tau=0.41, p=$ $0.42, \kappa=30, \mu=0.01, \gamma_{1}=4, \gamma_{2}=4.3, \rho=0.11, c=20$ and $(I(0), J(0), R(0), U(0))=$ $(0.01,0.02,0.11,0.30)$. To help visualizing the $I$ and $J$ components, we plotted $5 I$ and $5 J$ in the figure. The figure is generated by DDE23 with MATLAB.

2..10 Corollary. Let treatment be of fixed length and the mortality rate $\mu$ sufficiently small. Then, the parameters $0<p<1,0<\tau<1, \gamma_{1}>0$ and $\kappa>0$ can be chosen in such a way that the endemic equilibrium is unstable.

In fact, $p$ and $\kappa$ can be changed in such a way that a root of the characteristic equation crosses the imaginary axis.

Figure 2 (generated by DDE23 with MATLAB) illustrates that periodic solutions can occur.

Gamma-distributed treatment. We assume that the length of treatment (death neglected) is Gamma-distributed, i.e. $\mathcal{F}(a)=\int_{a}^{\infty} f(t) d t$ where $f$ is the Gamma-density

$$
f(t)=\frac{\alpha^{\nu} t^{\nu-1} e^{-\alpha t}}{\Gamma(\nu)}, \quad t \geq 0
$$

and $\Gamma$ the Gamma-function which makes $f$ a probability density. Here $\alpha$ is just a scaling parameter, but the parameter $\nu$ is essential. For $\nu=1$, the $\Gamma$-distribution is the exponential distribution. The expected length of the treatment, $D_{T}$, is $\nu / \alpha$ and the variance $V_{T}=\nu / \alpha^{2}$ [6, II.2]. By a substitution,

$$
\hat{f}(z)=\frac{1}{\Gamma(\nu)} \int_{0}^{\infty} \alpha^{\nu} t^{\nu-1} e^{-(\alpha+z) t} d t=\frac{\alpha^{\nu}}{(\alpha+z)^{\nu}} .
$$

So

$$
\hat{f}(\mu-i s)=\frac{\alpha^{\nu}}{(\alpha+\mu-i s)^{\nu}}=\left(\frac{\alpha(\alpha+\mu+i s)}{(\alpha+\mu)^{2}+s^{2}}\right)^{\nu} .
$$




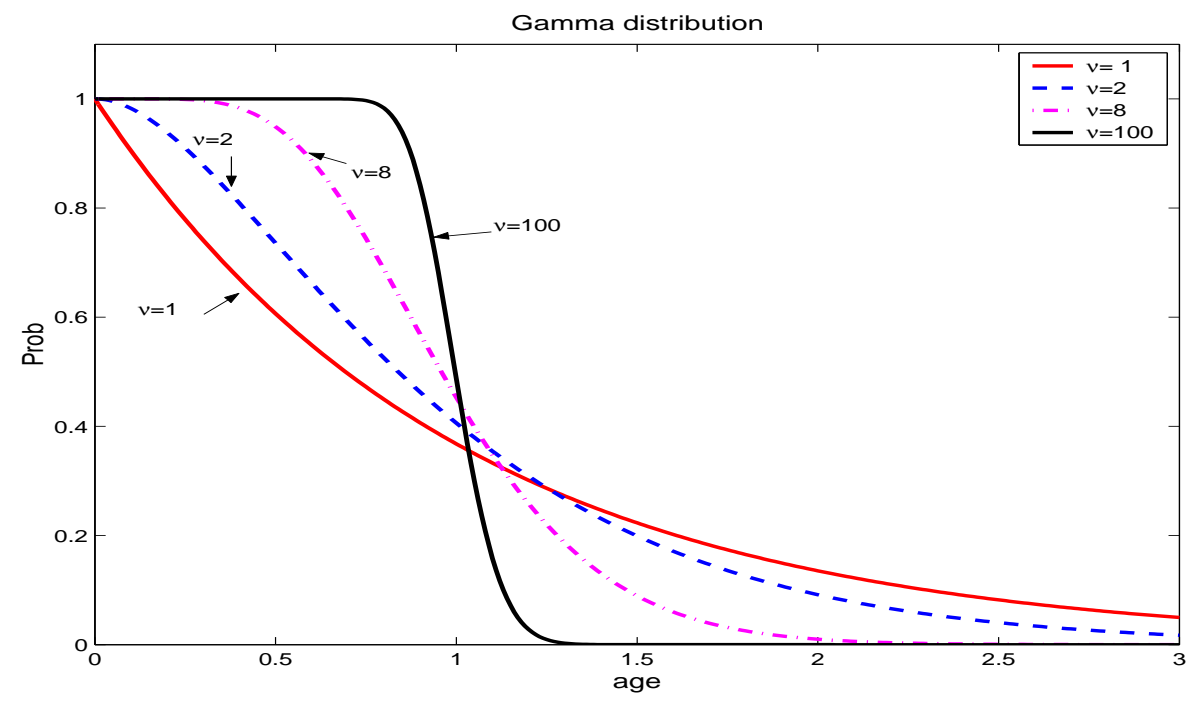

Figure 3: The $\Gamma$-distribution interpolates between treatment with fixed termination rate $(\nu=1)$ and treatment of fixed length $(\nu \rightarrow \infty)$. The various curves show the probability of still being treated at a certain treatment age for $\nu=1,2,8,100$. The expected length of treatment has been normalized to one in all cases. The figure is generated by plotting the built-in Gamma distribution function in MATLAB.

In polar coordinates, for $s>0, \hat{f}(\mu-i s)=r^{\nu} e^{\nu \phi}$ where $\phi \in(0, \pi / 2)$. Any $\phi \in(0, \pi / 2)$ is possible by choosing $s>0$ in a suitable way. So $\Im \hat{f}(\mu-i s) \geq 0$ if $\nu \leq 2$, while for $\nu>2$ the angle $\phi \in(0, \pi / 2)$ and thus $s>0$ can be chosen in such a way that $\Im \hat{f}(\mu-i s)<0$.

We obtain the following results from Theorem 2..3, Lemma 2..7, and Theorem 2..9. Notice that $\nu=D_{T}^{2} / V_{T}$.

2..11 Corollary. If the length of the treatment is $\Gamma$-distributed with $\nu \leq 2$, the endemic equilibrium is globally asymptotically stable. If $\nu>2$, after choosing $\mu>0$ sufficiently small the parameters $p, 0<\tau<1, \gamma_{1}$, and $\kappa$ can be chosen in such a way that the endemic equilibrium is unstable.

This result is independent of the parameter $\alpha$. If we set $\alpha=\nu / \sigma$, the average length of treatment, $D_{T}=\sigma$, is independent of $\nu$ while the variance $V_{T}=\frac{\sigma^{2}}{\nu}$ tends to 0 as $\nu \rightarrow \infty$. With this scaling, the Gamma-distribution interpolates between an exponentially distributed treatment for $\nu=1$ and a treatment of fixed length $\sigma$ for $\nu \rightarrow \infty$. See Figure 2..

\section{Discussion}

There is a longstanding interest in mathematical epidemiology in mechanisms that can induce undamped oscillations of the prevalence of an infectious disease. It would lead too far to even give a partial review in this paper, instead we refer to [7,9] and the references therein. One class of mechanisms is given by transfer of susceptible individuals into some other epidemiological class with subsequent return. Typically this return needs to involve some sort of delay in form 
of several stages or the involvement of some class-age-structure. One form of transfer out of the susceptible class is by infection itself. Undamped oscillations can occur if infected individuals become susceptible again in a delayed fashion by either going through several stages of recovery (at least three) $[10,15,17]$ or by an infection-age dependent $[14,13]$ or recovery-age dependent $[10,15]$ return.

A new way of transfer out of the susceptible class has been proposed in [8]. Susceptible individuals that have been exposed to the disease (had a contact with an infectious individuals) are treated with an antimicrobial drug which, if no infection has occurred, lowers their susceptibility. The uninfected treated individuals return into the susceptible class once treatment is terminated, presumably when it becomes evident that no infection has occurred in the first place.

While the main thrust in [8] is the possible development of drug-resistance, we are mainly interested in whether this new mechanism can lead to undamped oscillation if this form of antiviral treatment is used as a long-term strategy. We make the overly optimistic assumptions that treated uninfected individuals are not susceptible at all and that treated infected individuals are not infectious. (In [8, Tab.A1] it has been estimated that the susceptibility of treated uninfected individuals is 0.5 that of susceptible individuals and that the rate of infection by treated wild-type virus is 0.5 that by untreated wild-type virus.) These assumptions and some other simplifications are made in order to make an analytic investigation of the model possible which provides some clear statements when and when not undamped oscillations can occur. These statements can then serve as educated conjectures for more realistic models which can only be explored by numerical simulations or other computational tools. One such statement is that the disease dynamics tend to an equilibrium when the exposed uninfected individuals are not treated for too long, more precisely when

$$
\frac{\tau}{1-\tau} \tilde{D}_{T} \leq \frac{p}{1-p} \tilde{D}_{I}
$$

Here $\tilde{D}_{T}$ is the mean duration of treatment and $\tilde{D}_{I}$ the mean duration of the infectious period (for untreated infected individuals) with both durations taking natural death into account. Further $\tau$ denotes the probability of treatment and $p$ the probability that a contact between a susceptible and an infectious individual results in an infection (Corollary 2..6). Given the difficulty of identifying exposed individuals, it may be a reasonable assumption that $\tau$ is considerably smaller than $p$. In [8, Tab.A1], $p$ (1/c in their symbolism) has been assumed to be 0.5 in the absence of data. [ 8 , Tab.1] considers $\tau=0,0.01,0.05$ (and various levels of symptomatic prophylaxis, which is not incorporated in our model), while [8, Fig.4] covers a range of $\tau$ from 0 to 0.4. For the containment of an influenza pandemic, [12] assumes that $80 \%$ of all index cases could be ascertained and that these and their entire mixing groups (household, daycare center, playgroup, school) would receive prophylactic treatment. One would assume that exposed uninfected individuals are no longer treated once it becomes reasonably sure that they are uninfected indeed. [8] uses an average length of 6 days for the infectious period (two days asymptomatic and four days symptomatic) and an average length of treatment of uninfected exposed individuals of 3 days $(1 / \xi$ in Table A1). [12] considers treatment lengths of up to 1 week, 4 weeks, 6 weeks and 8 weeks and report that 6 weeks are almost as effective as 8 weeks but considerably more effective than 4 weeks in disease prevention. But this is for the containment of a pandemic, and the target unit is not a single exposed 
individual but the exposed mixing group of an index case. So one would cautiously conjecture that in typical endemic situations the average durations of prophylactic antimicrobial treatment are too short to give rise to undamped oscillations.

Our results do not only put restrictions on the average duration of the treatment, but also on its length distribution. For instance, if treatment is terminated at a fixed rate (leading to an exponential distribution of treatment length), the disease dynamics converge to an (disease-free or endemic) equilibrium even for very large treatment length averages (Corollary 2..5). More generally, if $\mathcal{F}(a)$, the probability of still being treated when treatment started $a$ time units ago, is a convex function of $a$, the disease dynamics converge towards an equilibrium (Corollary 2..4). The investigation of Gamma-distributed treatment lengths suggests that convergence to equilibrium occurs when the standard deviation is sufficiently large compared to the average length (Corollary 2..11). But we could not verify this conjecture for other treatment length distributions.

Conversely, the endemic equilibrium can be unstable for treatment lengths that are fixed (see Figure 2.) and (possibly unrealistically) large if the other model parameters are in suitable range (Corollary 2..10), and numerical simulations illustrate that undamped oscillations are possible in this case. The endemic equilibrium can also be unstable for Gamma-distributed treatment lengths where the standard deviation is small compared to the mean, under the same proviso as before (Corollary 2..11).

\section{A Proofs}

\section{A1. Transformation to a single equation}

We first write (1..2), (1..7) as a system of ordinary differential equations and a Volterra integral equation,

$$
\begin{aligned}
I^{\prime} & =(1-\tau) p \kappa I S-\left(\mu+\gamma_{1}\right) I, \\
J^{\prime} & =\tau p \kappa I S-\left(\mu+\gamma_{2}\right) J, \\
R^{\prime} & =\gamma_{1} I+\gamma_{2} J-(\mu+\rho) R \\
S & =1-U-I-J-R \\
U(t) & =\int_{0}^{t} \tau(1-p) \kappa I(t-s) S(t-s) \mathcal{G}(s) d s+\breve{U}(t), \\
\breve{U}(t) & =\int_{0}^{\infty} \frac{\mathcal{G}(a+t)}{\mathcal{G}(a)} u_{0}(a) d a .
\end{aligned}
$$

We add the initial conditions $I(0)=I_{0}, J(0)=J_{0}$, and $R(0)=R_{0}$. From the first equation,

$$
\kappa I S=\frac{1}{(1-\tau) p}\left(I^{\prime}+\left(\mu+\gamma_{1}\right) I\right) .
$$

We substitute this formula into the fifth equation and obtain

$$
U(t)=\phi \int_{0}^{t}\left(I^{\prime}(t-s)+\left(\gamma_{1}+\mu\right) I(t-s)\right) \mathcal{G}(s) d s+\breve{U}(t)
$$


with

$$
\phi=\frac{\tau(1-p)}{(1-\tau) p}
$$

We define

$$
\tilde{\mathcal{G}}(s)=\left\{\begin{array}{cc}
0, & s \leq 0, \\
\lim _{h \rightarrow 0+} \mathcal{G}(s-h), & s>0 .
\end{array}\right.
$$

Then $\tilde{\mathcal{G}}$ is left-continuous and

$$
U(t)=\phi \int_{0}^{t}\left(-\frac{d}{d s} I(t-s) \tilde{\mathcal{G}}(s)+\left(\gamma_{1}+\mu\right) I(t-s) \mathcal{G}(s)\right) d s+\breve{U}(t) .
$$

We integrate by parts using a Stieljes integral [16, Cor.B.11]. Since, by construction, $\tilde{\mathcal{G}}(0)=0$,

$$
U(t)=\phi\left(\int_{0}^{t} I(t-s) d \tilde{\mathcal{G}}(s)+\int_{0}^{t}\left(\gamma_{1}+\mu\right) I(t-s) \mathcal{G}(s) d s-I_{0} \tilde{\mathcal{G}}(t)\right)+\breve{U}(t) .
$$

Further, from the second equation in (A.1) and (A.2),

$$
J(t)=\frac{\tau}{1-\tau} \int_{0}^{t}\left(I^{\prime}(t-s)+\left(\mu+\gamma_{1}\right) I(t-s)\right) e^{-\left(\mu+\gamma_{2}\right) s} d s+J_{0} e^{-\left(\mu+\gamma_{2}\right) t} .
$$

In the following we define

$$
k_{j}(t)=e^{-\left(\mu+\gamma_{j}\right) t}, \quad j=1,2, \quad k_{3}(t)=e^{-(\mu+\rho) t}, \quad t \geq 0,
$$

and

$$
\tilde{k}_{j}(t)=0, \quad t \leq 0, \quad \tilde{k}_{j}(t)=k_{j}(t), \quad t>0 .
$$

Again we integrate by parts,

$$
\begin{aligned}
J(t)= & \frac{\tau}{1-\tau}\left(I(t)+\int_{0}^{t} I(t-s) k_{2}^{\prime}(s) d s+\left(\mu+\gamma_{1}\right) \int_{0}^{t} I(t-s) k_{2}(s) d s\right) \\
& +\breve{J}(t), \\
\breve{J}(t)= & \left(J_{0}-\frac{\tau}{1-\tau} I_{0}\right) k_{2}(t) .
\end{aligned}
$$

We will also use this equation in the following form

$$
J(t)=\frac{\tau}{1-\tau}\left(\int_{0}^{t} I(t-s) d \tilde{k}_{2}(s)+\left(\mu+\gamma_{1}\right) \int_{0}^{t} I(t-s) k_{2}(s) d s\right)+\breve{J}(t) .
$$

Finally we integrate the third equation in (A.1) using (A.8),

$$
R(t)=\int_{0}^{t}\left(\gamma_{1} I(t-s)+\gamma_{2} J(t-s)\right) k_{3}(s) d s+R_{0} k_{3}(t) .
$$


We substitute (A.10),

$$
R(t)=\int_{0}^{t} I(t-s) k(s) d s+\breve{R}(t)
$$

with

$$
\begin{aligned}
k(t)= & \gamma_{1} k_{3}(t)+\frac{\gamma_{2} \tau}{1-\tau} k_{3}(t)+\frac{\gamma_{2} \tau}{1-\tau}\left(k_{2}^{\prime} * k_{3}\right)(t) \\
& +\frac{\gamma_{2} \tau}{1-\tau}\left(\mu+\gamma_{1}\right)\left(k_{2} * k_{3}\right)(t)
\end{aligned}
$$

where $k_{2} * k_{3}$ is the convolution

$$
\left(k_{2} * k_{3}\right)(t)=\int_{0}^{t} k_{2}(t-s) k_{3}(s) d s \quad \text { etc. }
$$

and

$$
\breve{R}(t)=R_{0} k_{3}(t)+\left(J_{0}-\frac{\tau}{1-\tau} I_{0}\right)\left(k_{2} * k_{3}\right)(t) .
$$

We return to (A.1). We substitute (A.7), (A.11), and(A.12) into $S$ and obtain,

$$
\frac{I^{\prime}}{I}=(1-\tau) p \kappa(1-I-I \star \beta-\tilde{\zeta}(t))-\gamma_{1}-\mu
$$

with

$$
(I \star \beta)(t)=\int_{0}^{t} I(t-s) d \beta(s)
$$

and

$$
\begin{aligned}
\beta(s)= & \phi\left(\tilde{\mathcal{G}}(s)+\left(\gamma_{1}+\mu\right) \int_{0}^{s} \mathcal{G}(t) d t\right) \\
& +\frac{\tau}{1-\tau}\left(\tilde{k}_{2}+\left(\mu+\gamma_{1}\right) \int_{0}^{s} k_{2}(t) d t\right)+\int_{0}^{s} k(t) d t
\end{aligned}
$$

and

$$
\tilde{\zeta}(t)=\breve{U}(t)-\phi I_{0} \mathcal{G}(t)+\breve{J}(t)+\breve{R}(t), \quad t>0 .
$$

Let $I^{*}$ denote the infective component of endemic equilibrium. Then

$$
\frac{I^{\prime}}{I}=(1-\tau) p \kappa\left(I^{*}-I-\left(I^{*}-I\right) \star \beta\right)-\breve{w}
$$

where

$$
\begin{aligned}
\breve{w}(t)= & (1-\tau) p \kappa \tilde{\zeta}+\eta \int_{t}^{\infty} d \beta(r) \\
= & (1-\tau) p \kappa \tilde{\zeta}+\eta\left(\phi\left(\tilde{\mathcal{G}}(t)+\left(\gamma_{1}+\mu\right) \int_{t}^{\infty} \mathcal{G}(r) d r\right)\right. \\
& \left.+\frac{\tau}{1-\tau}\left(\tilde{k}_{2}+\left(\mu+\gamma_{1}\right) \int_{t}^{\infty} k_{2}(r) d r\right)+\int_{t}^{\infty} k(r) d r\right)
\end{aligned}
$$


with

$$
\eta=(1-\tau) p \kappa I^{*} .
$$

Set $v=I / I^{*}$. Then

$$
\frac{v^{\prime}}{v}=\eta(1-v-(1-v) \star \beta)-\breve{w} .
$$

Set

$$
w=\ln v \quad \text { and } \alpha=\beta+H_{0},
$$

where $H_{0}(s)=0$ for $s \leq 0$ and $H_{0}(s)=1$ for $s>0$. Then we obtain an equation of the form considered in [11],

$$
w^{\prime}+g(w) \star \alpha=\breve{w}
$$

with

$$
g(u)=\eta\left(e^{u}-1\right) .
$$

\section{Attractivity of the endemic equilibrium (abstract result)}

We check the assumptions of [11, Thm.3]. We notice that $u g(u)>u$ for $u \neq 0$ and $\breve{w}(t) \rightarrow 0$ as $t \rightarrow \infty$. Further $\alpha$ is of normalized bounded variation, zero on $(-\infty, 0]$, and left-continuous. For $t>0$, the total variation of $\alpha$ on $[t, \infty)$ can be estimated by

$$
\begin{aligned}
\int_{t}^{\infty}|d \alpha(s)| \leq & \phi\left(\tilde{\mathcal{G}}(t)+\int_{t}^{\infty}\left(\mu+\gamma_{1}\right) \mathcal{G}(s) d s\right) \\
& +\frac{\tau}{1-\tau}\left(k_{2}(t)+\left(\mu+\gamma_{1}\right) \int_{t}^{\infty} k_{2}(s) d s\right)+\int_{t}^{\infty} k(s) d s .
\end{aligned}
$$

So

$$
\begin{aligned}
& \int_{0}^{\infty}\left(\int_{t}^{\infty}|d \alpha(s)|\right) d t \\
\leq & \phi\left(\tilde{D}_{T}+\left(\mu+\gamma_{1}\right) \int_{0}^{\infty} s \mathcal{G}(s) d s\right) \\
& +\frac{\tau}{1-\tau}\left(\int_{0}^{\infty} k_{2}(t) d t+\left(\mu+\gamma_{1}\right) \int_{0}^{\infty} t k_{2}(t) d t\right)+\int_{0}^{\infty} t k(t) d t<\infty .
\end{aligned}
$$

Since $0 \leq I(t) \leq 1$ and we have uniform strong disease persistence, $w$ is bounded. It follows from $[11$, Thm.3] that $w(t) \rightarrow 0$ as $t \rightarrow \infty$, if

$$
\Re \int_{0}^{\infty} e^{i s t} d \alpha(t)>0 \quad \forall s \in \mathbb{R} .
$$

Since $w(t)=\ln I(t)-\ln I^{*}$ this implies that $I(t) \rightarrow I^{*}$ as $t \rightarrow \infty$.

Then $U(t) \rightarrow U^{*}$ as $t \rightarrow \infty$ by (A.7) and $J(t) \rightarrow J^{*}$ by (A.10). $R(t) \rightarrow R^{*}$ follows from the third equation in (1..2), $S(t) \rightarrow 1-I^{*}-J^{*}-R^{*}-U^{*}$ from the fourth equations in (1..2) and $\int_{0}^{\infty}\left|u(t, a)-u^{*}(a)\right| d a \rightarrow 0$ from (1..4) and (2..2).

We summarize. 
A.1 Proposition. Let (A.26) be satisfied. Then the endemic equilibrium attracts all solutions of (1..2), (1..7) with $I(0)>0$.

\section{A2. Local stability of the endemic equilibrium}

We claim that condition (A.26) implies local asymptotic stability.

Let $x_{0}=\left(u_{0}, I_{0}, J_{0}, R_{0}\right)$ and $(u(t, \cdot), I(t), J(t), R(t))=\Phi\left(t, x_{0}\right), t \geq 0$, be the solution of our model equations with initial data $x_{0}$. We have shown in [18] that $\Phi$ is a semiflow, i.e. $\Phi\left(t+r, x_{0}\right)=\Phi\left(t, \Phi\left(r, x_{0}\right)\right)$ for all $t, r \geq 0$. The endemic equilibrium $x^{*}$ satisfies $\Phi\left(t, x^{*}\right)=x^{*}$ for all $t \geq 0$. We write $\Phi\left(t, x_{0}\right)$ as a perturbation of $x^{*}, \Phi\left(t, x^{*}+\tilde{x}_{0}\right)=x^{*}+\tilde{x}(t)$. Since all ingredients of our model are continuously differentiable in the dependent variables, it follows that $\Phi\left(t, x_{0}\right)$ is differentiable in $x_{0}$ for every $t \geq 0$ and that the linear operators $\partial \Phi(t)$, the derivatives of $\Phi\left(t, x_{0}\right)$ in $x_{0}$ evaluated at $x_{0}=x^{*}$, form a $C_{0}$-semigroup of bounded linear operators. $\partial \Phi(t) x_{0}$ is the semiflow induced by the solutions of the linearization of system (1..2), (1..7) around the endemic equilibrium. If $\tilde{x}_{0}$ is sufficiently small, we have $\tilde{x}(t) \approx \partial \Phi(t) \tilde{x}(0)$. This suggests that the local stability of $x^{*}$ boils down to the stability of 0 for the linear expression $\tilde{x}(t)=\partial \Phi(t) \tilde{x}(0)$. Indeed, if follows from [4] that $x^{*}$ is locally asymptotically stable if $\|\partial \Phi(t)\| \rightarrow 0$ as $t \rightarrow \infty$. The latter is approached by considering $\tilde{x}$ in the form $\tilde{x}(t)=e^{z t} \bar{x}, \bar{x} \neq 0, z \in \mathbb{C}$. (Then $z$ is an eigenvalue of the infinitesimal generator of $\partial \Phi$ and $\bar{x}$ the associated eigenvector.) A similar consideration as in [18, App.B] shows that $\partial \Phi$ is a quasi-compact semigroup in the sense of [5, V.3], and Theorem 3.7 therein implies that $\|\partial \Phi(t)\| \rightarrow 0$ as $t \rightarrow \infty$ if and only if there are no $z \in \mathbb{C}$ and no $\bar{x}$ such that $\Re z \geq 0$ and $\partial \Phi(t) \bar{x}=e^{z t} \bar{x}$. See also [19, Prop.4.3].

In turn, if there exists some $\bar{x}$ and $z \in \mathbb{C}$ with $\Re z>0$ and $\partial \Phi(t) \bar{x}=e^{z t} \bar{x}$, then the endemic equilibrium $x^{*}$ is unstable ([4] and [5, V.3], Theorem 3.7, or [19, Prop.4.15]).

When we follow the procedure of reducing our system to a single differential-integral equation for $\partial \Phi$ rather than the semiflow $\Phi$ and look for $\bar{x}$ and $z \in \mathbb{C}$ with $\partial \Phi(t) \bar{x}=e^{\lambda z} \bar{x}$, we are lead to solutions $\tilde{w}(t)=e^{z t}$ of the following linearization of (A.24),

$$
\tilde{w}^{\prime}(t)+\eta \int_{0}^{\infty} \tilde{w}(t-s) d \alpha(s)=0 .
$$

We substitute $\tilde{w}(t)=e^{z t}$ and obtain the characteristic equation

$$
z+\eta \int_{0}^{\infty} e^{-z t} d \alpha(t)=0 .
$$

Condition (A.26) implies that (A.28) has no purely imaginary roots $z$. In order to see that there are no roots $z$ with positive real part, we introduce

$$
\alpha_{r}(t)=r \eta \alpha(t)-(1-r) e^{-t}, \quad 0 \leq r \leq 1,
$$

and we consider the characteristic equation

$$
0=z+\int_{0}^{\infty} e^{-z t} d \alpha_{r}(t)=z+r \eta \int_{0}^{\infty} e^{-z t} d \alpha(t)+\frac{1-r}{1+z} .
$$


We separate into real and imaginary part, $z=x+i y$,

$$
\begin{aligned}
& 0=x+\eta r \int_{0}^{\infty} e^{-x t} \cos (y t) d \alpha(t)+(1-r) \frac{1+x}{(1+x)^{2}+y^{2}} \\
& 0=y+\eta r \int_{0}^{\infty} e^{-x t} \sin (y t) d \alpha(t)-(1-r) \frac{y}{(1+x)^{2}+y^{2}} .
\end{aligned}
$$

For $r=0$, all roots have negative real part $x$. Suppose that (A.28) has a root with positive real part. This means, for $r=1$, there is a root with positive real part of (A.29). As we vary $r$ between 0 and 1, it follows from (A.30) that the roots remain bounded as long as they stay in the right half plane. By Rouché's theorem, they depend continuously on $r$. So, for some $r \in(0,1)$ there exists a root on the imaginary axis. For this $r$,

$$
0=r \eta \int_{0}^{\infty} \cos (y t) d \alpha(t)+\frac{1-r}{1+y^{2}}
$$

contradicting (A.26).

We summarize our findings and combine them with Proposition A.1.

A.2 Proposition. If (A.26) holds, the endemic equilibrium is globally asymptotically stable. The endemic equilibrium is unstable if the characteristic equation (A.28) has a solution $z$ with $\Re z>0$.

To make further progress we evaluate the Laplace-Stieltjes transforms of $\alpha$. By (A.23) and (A.18),

$$
\begin{aligned}
& \int_{0}^{\infty} e^{-z t} d \alpha(t) \\
= & \phi\left(1+\int_{0}^{\infty} e^{-z t} d \mathcal{G}(t)\right)+\phi\left(\gamma_{1}+\mu\right) \hat{\mathcal{G}}(z)+1 \\
& +\frac{\tau}{1-\tau}\left(1+\int_{0}^{\infty} e^{-z t} k_{2}^{\prime}(t) d t+\left(\mu+\gamma_{1}\right) \hat{k}_{2}(z)\right)+\hat{k}(z) .
\end{aligned}
$$

Here we have used the Laplace transform $\hat{k}_{2}(z)=\int_{0}^{\infty} e^{-z t} k_{2}(t) d t$. We integrate by parts,

$$
\begin{aligned}
& \int_{0}^{\infty} e^{-z t} d \alpha(t) \\
= & \phi\left(1+\int_{0}^{\infty} e^{-z t} d \mathcal{G}(t)\right)+\phi\left(\gamma_{1}+\mu\right) \hat{\mathcal{G}}(z)+1 \\
& +\frac{\tau}{1-\tau}\left(\mu+\gamma_{1}+z\right) \hat{k}_{2}(z)+\hat{k}(z) .
\end{aligned}
$$

From (A.13), since the Laplace transform turns a convolution into the product of the individual transforms,

$$
\hat{k}(z)=\gamma_{1} \hat{k}_{3}(z)+\frac{\gamma_{2} \tau}{1-\tau} \hat{k}_{3}(z)+\frac{\gamma_{2} \tau}{1-\tau} \widehat{k}_{2}^{\prime}(z) \hat{k}_{3}(z)+\frac{\gamma_{2} \tau}{1-\tau}\left(\mu+\gamma_{1}\right) \hat{k}_{2}(z) \hat{k}_{3}(z) .
$$


Since $\widehat{k_{2}^{\prime}}(z)=-1+z \hat{k}_{2}(z)$

$$
\hat{k}(z)=\gamma_{1} \hat{k}_{3}(z)+\frac{\gamma_{2} \tau}{1-\tau}\left(\mu+\gamma_{1}+z\right) \hat{k}_{2}(z) \hat{k}_{3}(z) .
$$

With this result,

$$
\begin{aligned}
& \int_{0}^{\infty} e^{-z t} d \alpha(t) \\
= & \phi\left(1+\int_{0}^{\infty} e^{-z t} d \mathcal{G}(t)\right)+\phi\left(\gamma_{1}+\mu\right) \hat{\mathcal{G}}(z)+1 \\
& +\gamma_{1} \hat{k}_{3}(z)+\frac{\tau}{1-\tau}\left(\mu+\gamma_{1}+z\right) \hat{k}_{2}(z)\left(1+\gamma_{2} \hat{k}_{3}(z)\right) .
\end{aligned}
$$

\section{A3. Global stability of the endemic equilibrium}

In order to check condition (A.26) to apply Proposition A.2, we evaluate the factor of $\frac{\tau}{1-\tau}$ in the last term of (A.33) for $z=-i s$. By (A.8) it equals

$$
\frac{\mu+\gamma_{1}-i s}{\mu+\gamma_{2}-i s}\left(1+\frac{\gamma_{2}}{\mu+\rho-i s}\right)
$$

The real part has the same sign as the real part of

$$
\left(\mu+\gamma_{1}-i s\right)\left(\mu+\gamma_{2}+i s\right)\left(1+\frac{\gamma_{2}(\mu+\rho+i s)}{(\mu+\rho)^{2}+s^{2}}\right)
$$

which has the same sign as the real part of

$$
\left(\left(\mu+\gamma_{1}\right)\left(\mu+\gamma_{2}\right)+s^{2}+i s\left(\gamma_{1}-\gamma_{2}\right)\right)\left((\mu+\rho)^{2}+s^{2}+\gamma_{2}(\mu+\rho)+i \gamma_{2} s\right)
$$

and thus the same sign as

$$
\left(\left(\mu+\gamma_{1}\right)\left(\mu+\gamma_{2}\right)+s^{2}\right)\left((\mu+\rho)^{2}+s^{2}+\gamma_{2}(\mu+\rho)\right)+\gamma_{2} s^{2}\left(\gamma_{2}-\gamma_{1}\right)
$$

When we expand this expression in terms of powers of $s$, the terms without $s$ and the factor of $s^{4}$ are positive. We collect the factors of $s^{2}$,

$$
\begin{aligned}
& \left(\mu+\gamma_{1}\right)\left(\mu+\gamma_{2}\right)+(\mu+\rho)^{2}+\gamma_{2}\left(\mu+\rho+\gamma_{2}-\gamma_{1}\right) \\
= & \left(\mu+\gamma_{1}\right) \mu+(\mu+\rho)^{2}+\gamma_{2}\left(2 \mu+\rho+\gamma_{2}\right)>0 .
\end{aligned}
$$

So, by (A.33),

$$
\Re \int_{0}^{\infty} e^{i s t} d \alpha(t)>\phi \Re\left(1+\int_{0}^{\infty} e^{i s t} d \mathcal{G}(t)+\left(\gamma_{1}+\mu\right) \int_{0}^{\infty} e^{i s t} \mathcal{G}(t) d t\right)+1 .
$$


Proof of Theorem 2..3. Since $\mathcal{G}$ is decreasing,

$$
\begin{aligned}
& \Re\left(1+\int_{0}^{\infty} e^{i s t} d \mathcal{G}(t)\right) \geq 1-\left|\int_{0}^{\infty} e^{i s t} d \mathcal{G}(t)\right| \\
\geq & 1-\int_{0}^{\infty}|d \mathcal{G}(t)|=1+\int_{0}^{\infty} d \mathcal{G}(t)=0 .
\end{aligned}
$$

So $\Re \int_{0}^{\infty} e^{i s t} d \alpha(t)>\phi\left(\gamma_{1}+\mu\right) \int_{0}^{\infty} e^{i s t} \mathcal{G}(t) d t+1$. We recall $\phi$, (A.4), and Theorem $2 . .3$ follows from Proposition A.2.

\section{A4. Some frequency domain results}

The following results are known, but may be not so widely and are proved for the reader's convenience.

A.3 Lemma. If $f: \mathbb{R}_{+} \rightarrow \mathbb{R}$ is decreasing and not constant a.e., then

$$
\int_{0}^{\infty} \sin (s t) f(t) d t>0, \quad s \geq 0,
$$

provided the integrals exist as improper integrals.

Proof. Since $f$ is decreasing, it is Borel measurable. The result is obviously true for $s=0$. If $s>0$, we can assume that $s=1$ by a change of variables. Since the integral exists as improper integral,

$$
\int_{0}^{\infty} \sin (t) f(t) d t=\lim _{n \rightarrow \infty} \int_{0}^{2 n \pi} \sin (t) f(t) d t
$$

By the periodicity of sine,

$$
\begin{aligned}
& \int_{0}^{2 n \pi} \sin (t) f(t) d t=\sum_{k=0}^{n-1} \int_{0}^{2 \pi} \sin (t) f(t+2 k \pi) d t \\
= & \sum_{k=0}^{n-1} \int_{0}^{\pi} \sin (t)(f(t+2 k \pi)-f(t+(2 k+1) \pi)) d t .
\end{aligned}
$$

Since $f$ is decreasing and $\sin t>0$ for $t \in(0, \pi)$, each term in the sum is non-negative. If $f$ is not constant a.e., at least one of the integrals is strictly positive for large enough $n$. The strict positivity is preserved in the limit because $\left(\int_{0}^{2 n \pi} \sin (t) f(t) d t\right)$ is an increasing sequence.

A.4 Lemma. Let $f: \mathbb{R}_{+} \rightarrow \mathbb{R}$ be convex and continuous. Then

$$
\int_{0}^{\infty} \cos (s t) f(t) d t \geq 0, \quad s>0,
$$

provided the integrals exist as improper integrals. These integrals are strictly positive if $f$ is strictly convex. 
Proof. Obviously the result holds if $s=0$. If $s>0$, we can assume $s=1$ after a change of variables. Since the integral exists as an improper integral,

$$
\int_{0}^{\infty} \cos (s t) f(t) d t=\lim _{n \rightarrow \infty} \int_{0}^{2 n \pi} \cos (s t) f(t) d t
$$

Since $f$ is convex, $f$ is absolutely continuous, $f^{\prime}$ is increasing [2, Prop.2.7.9], and

$$
\int_{0}^{2 n \pi} \cos (t) f(t) d t=\int_{0}^{2 n \pi} \sin (t)\left(-f^{\prime}(t)\right) d t .
$$

Since $-f^{\prime}$ is decreasing, the arguments in the proof of the previous lemma show that the last integral is non-negative. Notice that $f^{\prime}$ is not constant a.e., if $f$ is strictly convex.

\section{A5. Instability of the endemic equilibrium}

Proof of Theorem 2..9. Guided by Proposition A.2, we study the characteristic equation (A.28) which we rewrite as

$$
0=\frac{z}{\eta}+\int_{0}^{\infty} e^{-z t} d \alpha(t)
$$

with

$$
\eta=(1-\tau) p \kappa I^{*}
$$

By (A.33),

$$
\begin{aligned}
0= & \frac{z}{\eta}+\phi\left(1+\int_{0}^{\infty} e^{-z t} d \mathcal{G}(t)\right)+\phi\left(\gamma_{1}+\mu\right) \hat{\mathcal{G}}(z)+1 \\
& +\gamma_{1} \hat{k}_{3}(z)+\frac{\tau}{1-\tau}\left(\mu+\gamma_{1}+z\right) \hat{k}_{2}(z)\left(1+\gamma_{2} \hat{k}_{3}(z)\right) .
\end{aligned}
$$

This can be rewritten as

$$
\begin{aligned}
0= & \frac{\tilde{\tau} z}{\eta}+\tilde{p}\left(1+\int_{0}^{\infty} e^{-z t} d \mathcal{G}(t)+\left(\gamma_{1}+\mu\right) \hat{\mathcal{G}}(z)\right) \\
& +\tilde{\tau}\left(\frac{\gamma_{1}}{\mu+\rho+z}+1\right)+\left(\mu+\gamma_{1}+z\right) \hat{k}_{2}(z)\left(1+\gamma_{2} \hat{k}_{3}(z)\right)
\end{aligned}
$$

with

$$
\tilde{\tau}=\frac{1-\tau}{\tau}, \quad \tilde{p}=\frac{1-p}{p} .
$$

Let $\mu \geq 0, \gamma_{2} \geq 0$ and $\rho \geq 0$ be fixed, but arbitrary, $\mu+\gamma_{2}>0$ and $\mu+\rho>0$. We define

$$
\begin{aligned}
\Delta\left(z ; \gamma_{1}, \tilde{p}, \tilde{\tau}, \eta\right)= & \frac{\tilde{\tau} z}{\eta}+\tilde{p}\left(1+\int_{0}^{\infty} e^{-z t} d \mathcal{G}(t)+\left(\gamma_{1}+\mu\right) \hat{\mathcal{G}}(z)\right) \\
& +\tilde{\tau}\left(\frac{\gamma_{1}}{\mu+\rho+z}+1\right)+\tilde{\Delta}\left(z ; \gamma_{1}\right) \\
\tilde{\Delta}\left(z ; \gamma_{1}\right)= & \left(\mu+\gamma_{1}+z\right) \hat{k}_{2}(z)\left(1+\gamma_{2} \hat{k}_{3}(z)\right)
\end{aligned}
$$


We let $z=x+i y$ with $x, y \in \mathbb{R}$. Following [15], to solve $\Delta\left(z ; \gamma_{1}, \tilde{p}, \tilde{\tau}, \eta\right)=0$, we want to apply the implicit function theorem and so consider real and imaginary parts of $\Delta$,

$$
\begin{aligned}
\Re \Delta(x+i y)= & \frac{\tilde{\tau} x}{\eta}+\tilde{p}\left(1+\Re \int_{0}^{\infty} e^{-z t} d \mathcal{G}(t)+\left(\gamma_{1}+\mu\right) \Re \hat{\mathcal{G}}(z)\right) \\
& +\tilde{\tau}\left(\frac{\gamma_{1}(\mu+\rho+x)}{(\mu+\rho+x)^{2}+y^{2}}+1\right)+\Re \tilde{\Delta}\left(x+i y ; \gamma_{1}\right)
\end{aligned}
$$

and

$$
\begin{aligned}
\Im \Delta(x+i y)= & \frac{\tilde{\tau} y}{\eta}+\tilde{p}\left(\Im \int_{0}^{\infty} e^{-z t} d \mathcal{G}(t)+\left(\gamma_{1}+\mu\right) \Im \hat{\mathcal{G}}(z)\right) \\
& -\tilde{\tau} \frac{\gamma_{1} y}{(\mu+\rho+x)^{2}+y^{2}}+\Im \tilde{\Delta}\left(x+i y ; \gamma_{1}\right) .
\end{aligned}
$$

For $x=0$,

$$
\begin{aligned}
\Re \Delta(i y)= & \tilde{p}\left(1+\int_{0}^{\infty} \cos (y t) d \mathcal{G}(t)+\left(\gamma_{1}+\mu\right) \Re \hat{\mathcal{G}}(i y)\right) \\
& +\tilde{\tau}\left(\frac{\gamma_{1}(\mu+\rho)}{(\mu+\rho)^{2}+y^{2}}+1\right)+\Re \tilde{\Delta}\left(i y ; \gamma_{1}\right)
\end{aligned}
$$

and

$$
\begin{aligned}
\Im \Delta(i y)= & \frac{\tilde{\tau} y}{\eta}+\tilde{p}\left(-\int_{0}^{\infty} \sin (y t) d \mathcal{G}(t)-\left(\gamma_{1}+\mu\right) \int_{0}^{\infty} \sin (y t) \mathcal{G}(t) d t\right) \\
& -\tilde{\tau} \frac{\gamma_{1} y}{(\mu+\rho)^{2}+y^{2}}+\Im \tilde{\Delta}\left(i y ; \gamma_{1}\right) .
\end{aligned}
$$

As assumed in Theorem 2..9, let

$$
y>0, \quad \Re \hat{\mathcal{G}}(i y)=\int_{0}^{\infty} \cos (y t) \mathcal{G}(t) d t<0 .
$$

By choosing $\gamma_{1}>0$ large enough we can achieve that

$$
1+\int_{0}^{\infty} \cos (y s) d \mathcal{G}(s)+\left(\gamma_{1}+\mu\right) \Re \hat{\mathcal{G}}(i y) d s<0
$$

and

$$
-\int_{0}^{\infty} \sin (y s) d \mathcal{G}(s)-\left(\gamma_{1}+\mu\right) \int_{0}^{\infty} \sin (y s) \mathcal{G}(s) d s<0 .
$$

By Lemma A.3, $\int_{0}^{\infty} \sin (y s) \mathcal{G}(s) d s>0$ because $\mathcal{G}$ is decreasing and not constant. We choose $\tilde{\tau}>0$ so large (by choosing $\tau>0$ in (A.35) small enough) that

$$
-\tilde{\tau} \frac{\gamma_{1} y}{(\mu+\rho)^{2}+y^{2}}+\Im \tilde{\Delta}\left(i y ; \gamma_{1}\right)<0 .
$$


A similar consideration as in Section A3. shows that $\Re \tilde{\Delta}\left(i y ; \gamma_{1}\right)>0$. We can now choose $\tilde{p}=$ $\tilde{p}_{0}>0$ such that $\Re \Delta\left(i y ; \breve{\gamma}_{1}, \tilde{p}_{0}, \tilde{\tau}, \eta\right)=0$. Observe from (A.40) that $\Im \Delta\left(i y, \gamma_{1}, \tilde{p}_{0}, \tilde{\tau}, \eta\right)-\frac{\tilde{\tau} y}{\eta}$ is a negative constant that does not depend on $\eta>0$ and from (A.39) that $\Re \Delta\left(i y ; \breve{\gamma}_{1}, \tilde{p}_{0}, \tilde{\tau}, \eta\right)$ does not depend on $\eta$. Finally we can choose $\eta=\eta_{0}>0$ such that $\Im \Delta\left(i y ; \gamma_{1}, \tilde{p}_{0}, \tilde{\tau}, \eta_{0}\right)=0$. We define

$$
F(x ; \tilde{p}, \eta)=\left(\begin{array}{l}
\Re \Delta\left(x+i y, \gamma_{1}, \tilde{p}, \tilde{\tau}, \eta\right) \\
\Im \Delta\left(x+i y, \gamma_{1}, \tilde{p}, \tilde{\tau}, \eta\right)
\end{array}\right)
$$

Then $F\left(0, \tilde{p}_{0}, \eta_{0}\right)=0$. The partial derivative $\frac{\partial F_{1}}{\partial \tilde{p}}(0, \tilde{p}, \eta)$ is the left hand side of (A.42) and negative, while $\frac{\partial F_{1}}{\partial \eta}=0$. Further $\frac{\partial F_{2}}{\partial \eta}<0$. So the Jacobian matrix of $F$ with respect to $(\tilde{p}, \eta)$, evaluated at $\left(0, \tilde{p}_{0}, \eta_{0}\right)$, is non-zero. By the implicit function theorem, there exist an open interval $W$ and an open set $V$ such that $0 \subseteq W \subseteq \mathbb{R}$ and $\left(\tilde{p}_{0}, \eta_{0}\right) \in V \subseteq \mathbb{R}^{2}$ such that, for every $x \in W$, $z=x+i y$ is a root of the characteristic equation for some $(\tilde{p}, \eta) \in V$. More precisely, there exists a function $f: W \rightarrow V$ such that, whenever $x \in W$ and $(\tilde{p}, \eta) \in V$, then $F(x ; \tilde{p}, \eta)=0$ if and only if $(\tilde{p}, \eta)=f(x)$. Since $F$ is analytic, so is $f$ [3, Ch.2 Thm.2.3].

In particular one can obtain roots with positive and with negative real parts by choosing $(\tilde{p}, \eta) \in$ $V$ accordingly.

We still need to show that the compound parameter $\eta$, given by (A.22), can be chosen independently of $\tilde{p}=\frac{p}{1-p}, \tilde{\tau}=\frac{\tau}{1-\tau}$, and $\gamma_{1}$ as we did above. By (A.16),

$$
(1-\tau) p \kappa\left(1-I^{*}-I^{*} \int_{0}^{\infty} d \beta(t)\right)=\mu+\gamma_{1} .
$$

By (A.23),

$$
\eta=\frac{(1-\tau) p \kappa-\mu-\gamma_{1}}{\int_{0}^{\infty} d \alpha(t)}
$$

By (A.33), $\int_{0}^{\infty} d \alpha(t)>0$ and does not depend on $\kappa$. So, by varying $\kappa$ (under the restriction that $\mathcal{R}_{0}>1$ ), we can choose every $\eta>0$ independently of $p, \tau$ (and so $\tilde{\tau}$ ) and $\gamma_{1}$. This completes the proof of Theorem 2..9.

\section{Acknowledgments}

This research was partially supported by NSF grants DMS-0314529 (HRT) and DMS-0436341 and DMS/NIGMS-0342388 (YK and AT). Correspondence should be directed to Horst Thieme.

\section{References}

[1] M.E. Alexander, C.S. Bowman, Z. Feng, M. Gardam, S.M. Moghadas, G. Röst, J. Wu, P.Yan. Emergence of drug resistance: implications for antiviral control of pandemic influenza,. Proc. Royal Soc. B, 274, (2007), 1675-1684. 
[2] W. Arendt, C.J.K. Batty, M. Hieber, F. Neubrander. Vector-valued Laplace transforms and Cauchy problems. Birkhäuser, Basel, 2001.

[3] S.N. Chow, J.K. Hale. Methods of bifurcation theory. Springer, New York, 1982.

[4] W. Desch, W. Schappacher, Linearized stability for nonlinear semigroups. In: Differential Equations in Banach Spaces. (A. Favini, E. Obrecht, eds.), 61-73, Springer, Heidelberg, 1986.

[5] K.-J. Engel, R. Nagel. One-parameter semigroups for linear evolution equations. Springer, New York, 2000.

[6] W. Feller. An Introduction to probability theory and its applications. Vol. II, Wiley Eastern Limited, New Delhi, 1977.

[7] Z. Feng, H.R. Thieme. Endemic models with arbitrarily distributed periods of infection. II. Fast disease dynamics and permanent recovery. SIAM J. Appl. Math., 61 (2000), 983-1012.

[8] N.M. Ferguson, S. Mallett, H. Jackson, N. Roberts, P. Ward. A population-dynamic model for evaluating the potential spread of drug-resistant influenza virus infections during community-based use of antivirals. J. Antimicrobial Chemotherapy, 51, (2003), 977-990.

[9] H.W. Hethcote, S.A. Levin. Periodicity in epidemiological models. In: Applied Mathematicl Ecology. (S.A. Levin, T.G. Hallam, L.J. Gross, editors), 193-211, Springer, Berlin Heidelberg, 1989.

[10] H.W. Hethcote, H.W. Stech, P. van den Driessche, Nonlinear oscillations in epidemic models. SIAM J. Appl. Math., 40, (1981), 1-9.

[11] S.-O. Londen. On the variation of the solution of a nonlinear integral equation. J. Math. Anal. Appl., 52, (1975), 430-449.

[12] I.M. Longini, M.E. Halloran, A. Nizam, Y. Yang. Containing pandemic influenza with antiviral agents. Amer. J. Epid., 159, (2004), 623-633.

[13] M. Martcheva, S.S. Pilyugin. The role of infection in multidisease dynamics. SIAM J. Appl. Math., 66, (2006), 843-872.

[14] J.A.J. Metz, O. Diekmann. The dynamics of physiologically structured populations. Springer, Berlin Heidelberg, 1986.

[15] H.W. Stech, M. Williams. Stability in a class of cyclic epidemic models with delay. J. Math. Biol., 11, (1981), 95-103.

[16] H.R. Thieme. Mathematics in population biology. Princeton University Press, Princeton, 2003. 
[17] H.R. Thieme, P. van den Driessche. Global stability in cyclic epidemic models with disease fatalities. Fields Institute Communications, 21, (1999), 459-472.

[18] H.R. Thieme, A. Tridane, Y. Kuang. An epidemic model with post-contact prophylaxis of distributed length. I. Thresholds for disease persistence and extinction. J. Biol. Dynamics, 2, (2008), 221-239.

[19] G.F. Webb. Theory of nonlinear age-dependent population dynamics. Marcel Dekker, New York, 1985. 